\title{
Universidad en el siglo XXI: ¿extensión, responsabilidad social universitaria, vínculo con el medio o conocimiento para el desarrollo de los territorios?
}

\author{
University in the 21st century: extension, university social responsibility, link with the \\ means or knowledge for the development of the territories?
}

\author{
Guillermo Williamson Castro* \\ Néstor Contreras Fuentes** \\ Me gustan los estudiantes \\ Porque son la levadura \\ Del pan que saldrá del horno \\ Con toda su sabrosura \\ Para la boca del pobre \\ Que come con amargura. ${ }^{1}$
}

\section{Resumen}

La universidad en Chile enfrenta enormes desafíos producto de la crítica de movimientos sociales, indígenas, académicos, empresariales y de los partidos políticos debido a su distanciamiento factual de los grandes problemas que enfrentan los territorios y sus procesos de desarrollo. La búsqueda de respuestas a esos requerimientos, justos y democráticos, en el marco de una economía neoliberal, lleva a reflexionar críticamente sobre las relaciones entre la universidad y el territorio según las concepciones dominantes en diversos momentos o instancias en el país: extensión, responsabilidad social universitaria, vínculo con el medio. La reflexión se inicia con un análisis contextual de la realidad universitaria chilena respecto de la temática, luego se discute brevemente la cuestión del territorio para continuar revisando, de modo crítico, las concepciones que han marcado las interacciones con la sociedad desde 1990 a la fecha, para terminar presentando la propuesta actual: vínculo con el Medio, que se instala desde el estado -aún de modo inicial- como componente clave en los sistemas de aseguramiento de la calidad universitaria, particularmente en los procesos de acreditación. Finalmente plantea algunas conclusiones y realiza algunas propuestas desde una concepción de comunicación para el conocimiento orientado al desarrollo de los territorios.

Palabras-claves: Extensión. Responsabilidad social universitaria. Territorio. Universidad. Vínculo con el Medio.

Recebido em 16/02/2018 - Aprovado em 10/06/2018

http://dx.doi.org/10.5335/rep.v25i3.8566

Doctor en Educación. Profesor Asociado Departamento de Educación. Director Magister en Desarrollo Humano Local y Regional Universidad de La Frontera. Miembro de Comité de Área de Educación Parvularia, Básica y Diferencial. Comisión Nacional de Acreditación (CNA-Chile). E-mail: guillermo.williamson@ufrontera.cl

** Trabajador Social. Magister en Desarrollo Humano Local y Regional. Coordinador de Formación Continua y Profesional Dirección de Vinculación con el Medio, Facultad de Educación, Ciencias Sociales y Humanidades, Universidad de La Frontera. E-mail: nestor.contreras@ufrontera.cl 


\title{
Abstract
}

\begin{abstract}
Universities in Chile face great challenges as a result of criticism from social indigenous academic business movements and political parties due to their factual distancing from the major issues that territories and development processes meet. The search for answers to all those just and democratic requirements within the frame of a neoliberal economy leads to reflect critically on the relationship between the university and the territory according to dominant conceptions at different times or Instances in the country: extension, university's social responsibility, link with the environment. The reflection begins with a contextual analysis of the Chilean university reality, according to the subject; then the question of the territory is briefly discussed to continue reviewing, critically, the conceptions that have marked the interactions with society from 1990 to date, ending by presenting the current proposal: Link with the Environment, which is set by the state -even ina a beginning mode- as a key component in university quality assurance systems, particularly in the accreditation processes. Finally it raises some conclusions and makes some proposals from a conception of communication for knowledge oriented to the development of territories.
\end{abstract}

Keywords: Extension. Link with the environment. Territory. University. University social responsibility.

\section{Introducción}

Hace 100 años la lucha por una universidad democrática, de calidad en la producción y difusión del conocimiento, comprometida con la transformación de la sociedad y la justicia social, que como casi siempre ha sido, la encabezaron los estudiantes - jardín de nuestras alegrías en versos de Violeta Parra - fue por participación en el gobierno y gestión universitaria. En Chile la Reforma Universitaria de 1967 consiguió esa conquista - iniciada, como la de Córdoba, en Universidades Católicas - pero la Dictadura Cívico-militar (1973-1980) la hizo retroceder retornando al autoritarismo de los gobiernos y hasta hoy - con algunas iniciativas tibias - aún no se reconquistan los avances de 1918 y de 1967. Esa es un movimiento que en Chile sigue pendiente y activo.

En 1981, la dictadura cívico-militar cambia radical y autoritariamente el sistema universitario en un contexto de cambio societal: el contexto de las transformaciones económicas de un modelo capitalista de sustitución de importaciones a uno neoliberal, en educación de una concepción de estado docente a una de estado subsidiario, en lo político el paso de una institucionalidad de democracia liberal a una democracia protegida, de un sistema de educación superior nacional a uno descentralizado, de una universidad comprometida con lo público a una comprometida con el mercado. Es el tiempo en que se establece el actual sistema universitario, que, pese a los ya casi 30 años de regímenes políticos reformistas en contextos de democracia protegida, se ha mantenido casi igual.

El modelo de economía de mercado de carácter neoliberal se expresó en la universidad estableciendo diversos mecanismos de competencia entre ellas, con el estado financiando un sector privado de empresas que extraía ganancias privadas 
de los aportes estatales a la educación, multiplicando la oferta profesional independientemente de sus posibilidades de inserción laboral, con un amplio sector de instituciones de educación superior de muy baja calidad y sin control o regulación estatal o social efectiva.

La desigualdad de calidad de la formación se profundizó en un contexto de masificación de la oferta de matrícula: al año 2010, 30 años después de que se establece el actual sistema universitario (1981) 900.936 personas estudiaban en educación superior, de los cuales 562.583 en el sistema universitario (JIMÉNEZ; LAGOS, 2011, p. 16). La privatización del sistema universitario, al permitirse el lucro en la educación (entendido como extracción de recursos públicos para el beneficio privado de algunos grupos sostenedores de las instituciones), perjudicó la calidad media del sistema y estableció profundas desigualdades en el sistema que afectaron a los sectores mayoritarios de la población sin contribuir al desarrollo del país sino desde: la formación de personas de pre a postgrado y educación continua en la última década, la generación de proyectos de desarrollo asociados a fundaciones de empresas o empresas directamente que tienen escasa cobertura territorial, apoyo al conocimiento necesario para la reproducción del capital en sectores específicos de la producción, medio de marketing de empresas para justificar su Responsabilidad Social Empresarial (RSE). Universidades estatales, algunas católicas y comunitarias y ciertas unidades o académicos individuales han buscado medios de influir o asociarse a los procesos de desarrollo local o regional y nacional, sin embargo, el sistema universitario se ha centrado en sí mismo, en alcanzar estándares y posiciones en los rankings para competir por las matrículas y consecuentemente por los recursos, dado el sistema de financiamiento que coloca los aportes privados personales como los principales para la subsistencia y los estatales como complemento (se calcula que el estado financia aproximadamente el $17 \%$ del presupuesto de las universidades estatales, la parte mayoritaria se debe a aportes de los estudiantes y el resto se debe lograr a través de ventas de servicios, overhead sobre proyectos ganados por los académicos, recursos asociados a la productividad en concursos de investigaciones y publicaciones y en el caso de algunas universidades, a donaciones).

Jiménez y Lagos (2011) hace una completa tipología de las universidades combinando investigación, docencia y acreditación en la cual, sin embargo, no es el compromiso con la sociedad o el vínculo entre conocimiento y desarrollo de la sociedad una dimensión que haga alguna diferencia entre las instituciones.

Después de décadas de olvido (y desprecio) del compromiso de la Universidad con el pueblo, con toda la ambigüedad del concepto (BADIOU, 2014), con el desarrollo, con la transformación de la sociedad, la reforma de la educación superior 
(a partir del 2015) ha privilegiado el denominado "Vínculo con el Medio" y lo ha colocado como una dimensión central en los procesos de aseguramiento de la calidad de la educación y su correspondiente acreditación. ¿Qué significa? ¿Cuánto de "sinceridad" es el esfuerzo del sistema por establecer ese vínculo? ¿Cambia algo en la concepción universitaria de orientación pública o es un nuevo disfraz de la privatización del sistema? Al fin... ¿desde una perspectiva crítica de qué se trata la Vinculación con el Medio en el Chile de hoy y del mañana?

Este artículo no se fundamenta en una investigación particular. Emerge como una sistematización, reflexiva y crítica, desde los aprendizajes generados en la tarea académica de proyección del quehacer en/de una universidad pública y estatal, durante décadas, a través de un conjunto de proyectos encabezados por el autor del presente trabajo en muchas dimensiones del quehacer universitario: educomunicación y radios comunitarias, educación intercultural bilingüe y educación multicultural, investigación en sala de clases, educación rural, educación de jóvenes y adultos, desarrollo humano local y regional, formación ciudadana, cooperativismo, formación inicial docente. ${ }^{2}$ Una parte importante de este quehacer se ha difundido a través de diversas publicaciones académicas. Para ser fieles al principio que orienta esta reflexión: el mundo puede ser un gran territorio lleno de vida transformadas en experiencias participativas y liberadoras que generan conocimiento desde la inserción activa en su construcción y transformación. Los participantes de los procesos educativos pueden ser investigadores, experimentadores, actores y protagonistas de la producción de conocimiento que emerge desde sus propias prácticas, praxis, reflexiones y acciones políticas, culturales, sociales de transformación del mundo.

\section{La universidad del siglo XX: el compromiso de la universidad con el pueblo de} Córdoba a Chile

Una brevísima reflexión contextual que es necesaria. La educación universitaria pública, estatal o de colaboración con el estado, sustentada en el servicio a la sociedad y sin fines de lucro (esto, aunque parezca extraño en nuestro continente para las universidades públicas, en el Chile neoliberal, es necesario explicitarlo) ha tenido diversos momentos de transformaciones significativas: desde la reforma de Córdoba en Argentina que democratiza la universidad abriendo la elección de autoridades a los estudiantes y afirma la vocación pública al servicio del pueblo. Tuvo proyecciones en Chile sin efectos similares hasta cuando en 1967 se produce la Reforma Universitaria que recoge las rupturas de límites autoritarios y arcaicos y que recién se conquistan, con una escasa duración hasta el golpe cívico-militar 
de 1973 cuando se instala un sistema autoritario, vertical y separado del pueblo que, en la práctica se proyecta hasta hoy. En Chile las universidades, en cuanto instituciones, tuvieron una escasa primavera democrática: entre agosto de $1967 \mathrm{y}$ septiembre de 1973, apenas 6 años; el resto de los años, desde el inicio del Siglo XX hasta la segunda década del XXI se ha mantenido bajo regímenes autoritarios o semi-democráticos. Puede decirse lo mismo respecto de las acciones de compromiso creador y transformador de la universidad pública con la sociedad.

En Chile hablar de universidad estatal y pública en la última centuria es hablar de una institución vertical, jerarquizada, semi-democrática, separada de la sociedad mayoritaria, sus intereses y desafíos de desarrollo. Sólo entre 1967 y 1973 se puede hablar de una Universidad profundamente arraigada en un proyecto de desarrollo nacional, asociado a la democratización de la sociedad y con auténtica democracia formal en su interior. Podemos decir que sólo en dicho periodo las comunidades universitarias de las universidades estatales y católicas ejercieron efectivamente una ciudadanía universitaria. En otros momentos no lo ha vivido, no vive nadie, sólo lo ha ejercido el estamento académico o parte de él ha experimentado alguna potestad democrática.

En conclusión: ¿es posible una universidad comprometida con la sociedad si en su interior no es democrática? Ya sabemos que en los regímenes dictatoriales esta es una pregunta sin sentido, pues la democracia es un valor expulsado de la sociedad, pero en regímenes políticos y universitarios democráticos, restringidos o decadentes, la cuestión asume vital importancia y la respuesta sólo puede ser una: no hay posibilidad. Por ello, porque la sociedad no existe para una universidad elitista, jerárquica, semidemocrática o no democrática, o si existe, debe estar muy enmarcada en sus límites de modo que no ultrapase las barreras que la separa y distingue del poder y las decisiones internas. Sin democracia interna no puede haber una verdadera vinculación con la sociedad, al menos desde lo institucional, pues académicos militantes, progresistas, comprometidos si pueden actuar de motu proprio en esa dirección, sin embargo, no consiguen alterar el orden vigente que mira el conocimiento, la ciencia, incluso la formación de personas hacia su interior o el mundo académico o de la ciencia y no el de la sociedad y el desarrollo que pasan a ser simples objetos de investigación o justificativa para experimentar con ellos. Sólo la acción política al interior de las universidades puede cambiar su sentido, su dirección, su organización. La educación es política señalaba Paulo Freire (2001), ambas dimensiones de la vida se funden en un mismo acto pedagógico pues comparten una visión de sociedad, de persona humana, de valores, de utopía. La universidad debe ser un espacio político plural y democrático ${ }^{3}$ de debate académico, de enfoques teóricos y paradigmas de conocimiento, pero debe tener dos objetivos complementarios en relación a un mismo propósito: la búsqueda honesta de la verdad y la puesta al servicio de una mejor sociedad de las respuestas encontradas. 
La universidad debe establecer una profunda reflexión, contextualizada al Siglo XXI respondiendo a las preguntas: ¿Ciencia para qué? ¿Formación Científica para qué? ¿Ciencia para quién? (NOGUEIRA, 2000), preguntas que emergen desde los paradigmas universales de la ciencia surgida desde lo que se denomina "occidente", pero además se hace necesario retroceder un paso y preguntarse, en un mundo en el que emerge el conocimiento ancestral indígena, el pensamiento "alternativo", la cultura popular y rural: ¿Qué ciencia? ¿Qué es la Ciencia? Son preguntas que ya se han hecho presentes a lo largo de toda la historia, sin embargo en cada época emergen nuevos conceptos científicos, disciplinarios o metodológicos así como innovadoras teorías y prácticas de producción y difusión de conocimientos, como la etnociencia, la etnomatemática, la investigación acción participativa, la medicina alternativa o intercultural, la producción y uso de hierbas medicinales. La emergencia desde el mundo externo a la universidad de un conjunto de saberes, conocimientos, epistemologías, cosmovisiones, prácticas sociales de observación e investigación, va enseñando que en la realidad el conocimiento social, popular, indígena de los territorios empieza, lentamente, a permear la universidad y espera de ésta, no una resistencia o un acto de refuerzo a su forma de ser autoritaria y orgullosa de su conocimiento sino un acto de reconocimiento al derecho de todos los seres humanos de ser productores de saberes, al saber acumulado en la vida y memoria cognitiva, corporal y patrimonial de las personas, en la naturaleza, en los movimientos sociales, populares e indígenas: en los territorios. Y el derecho de esa población, de ese territorio a que su conocimiento sea considerado como fuente válida de saberes por la universidad pública y estatal; los territorios y quienes los habitan de diversas formas tienen derecho a que su conocimiento acumulado a lo largo de cientos y miles de años se entregue y haga carne (curriculum, pedagogía, didáctica) en la formación de los profesionales, en la organización de los saberes como fuentes de conocimiento y metodologías de investigación y en los propósitos del trabajo académico, para que éste sirva efectivamente a la construcción de una sociedad justa, libre, democrática, no sólo en la institucionalidad política, sino en la valoración de todos los saberes y sus productores. No se trata de sobrevalorar o despreciar el avance del conocimiento científico, organizado como teorías o explicaciones, de la Humanidad, pero tampoco sobrevalorar o despreciar el avance del conocimiento tradicional, sistematizado como saber, de la Humanidad. No puede desconocerse que los paradigmas estructurados, las teorías, los métodos y tecnologías se desarrollan en contextos históricos dinámicos donde la cultura es dinámica, hoy más que nunca en la historia humana. La ciencia, en realidad combina paradigmas metodológicamente estructurados con dinamismos de la cultura en transformación (NOGUEIRA, 2000, p. 99): no hay ciencia fuera de la historia de los territorios y 
de su época, pero tampoco la hay fuera de la naturaleza y del saber acumulado por la Humanidad, que no está constituida sólo por los científicos o sabios o teólogos o poderosos, sino también por los humildes, los sencillos, los sabios del mundo cotidiano. Sacks (2002) hace una hermosa valoración del aporte de "los aficionados" a la investigación aplicada, a la ciencia, por ejemplo, a las flores y helechos:

En el trabajo de campo, en la ciencia aplicada, los aficionados siguen haciendo grandes contribuciones, como lo han hecho en el transcurso de los siglos [...]. Eso es lo que tienen los aficionados, en el mejor sentido de la palabra: pasión, amor por su tema y la experiencia acumulada, con frecuencia a lo largo de toda una vida de aguda observación en su campo de estudio [...] (SACKS, 2002, p. 32-33).

Esta visión destruye una perspectiva extensionista (de la universidad a partes de la sociedad, del investigador al consumidor de sus saberes) y plantea un enfoque del quehacer universitario centrado en la comunicación y en la responsabilidad cooperativa con la construcción de los territorios.

Cambiar el paradigma dominante que desprecia el saber "no científico" en el sentido "occidental y moderno" es una acción política, en la sociedad y en la universidad. Por ejemplo, en un mundo globalizado multilingüe y multicultural, en que emergen los pueblos indígenas en lucha por su reconocimiento o en el que los migrantes transnacionales y refugiados deambulan por el mundo, la universidad no se interculturaliza (WILLIAMSON, 2017) pues ello la obligaría a replantear sus paradigmas científicos, sus opciones de gestión y participación, el sentido de sí misma, autocriticarse en sus descubrimientos. Democratizar, interculturalizar y descolonizar la universidad (MATO, 2017) es una condición necesaria para que en la sociedad global y particularmente en los territorios con alta población indígena o afrodescendiente sea posible establecer un nuevo trato, un nuevo acuerdo, una nueva relación entre la universidad y el territorio. La ruptura con la dicotomía positivista de la tensión modernidad-tradición, por la eliminación de esta última, es una obligación de una concepción moderna, transformadora y científica de la universidad, adecuada a los cambios de una sociedad democrática, global, multicultural.

Vale la pena recordar el llamado de Córdoba en relación a terminar con un modelo de universidad vertical, jerarquizada, elitista, separada de la sociedad:

Hombres de una república libre, acabamos de romper la última cadena que en pleno siglo $\mathrm{XX}$ nos ataba a la antigua dominación monárquica y monástica. Hemos resuelto llamar a todas las cosas por el nombre que tienen. Córdoba se redime. Desde hoy contamos para el país una vergüenza menos y una libertad más. Los dolores que nos quedan son las libertades que nos faltan. Creemos no equivocarnos, las resonancias del corazón nos lo advierten: estamos pisando sobre una revolución, estamos viviendo una hora americana (MANIFIESTO..., 1918, p. 5). 
¿Cuál era esa revolución?: una universidad democrática en que la comunidad - incluidos los estudiantes - elige sus propias autoridades y que se compromete con el desarrollo del país y de la sociedad desde la perspectiva de las mayorías. La revolución aún pendiente es la de contar con universidades públicas (estatales, comunitarias y católicas gratuitas) capaces de estudiar y proyectar la dinámica de la sociedad y del mundo, sus tendencias de desarrollo, las transformaciones del territorio para entenderlos, adelantarse a sus crisis y errores, criticar sus fallas y distorsiones, encontrar soluciones a los problemas y desafíos que se presentan históricamente. Esta cuestión obliga a recuperar el carácter productor de bienes públicos que debe tener la universidad pública, aunque parezca contradictorio: hoy la privatización del sistema educacional - de mercado - ha afectado a las universidades estatales que deben prácticamente autofinanciarse para cumplir sus mandatos sociales y científicos. Una comunidad sin universidades públicas productoras de bienes públicos ¿puede comprometerse con la transformación social, democracia y justicia social? Claramente no, aunque parezca a la percepción del sentido común. Su aporte específico objetivo es mínimo, salvo en la cuantificación expansiva de formación de "capital humano" (disociada del mercado laboral) pero es limitada en sus aportes a la cultura como producción humana de sentidos, hechos y patrimonio físico e intangible. La sociedad civil, las organizaciones populares y movimientos sociales, observan la universidad como lejana en relación al mejoramiento de sus condiciones y comprensiones de vida: no esperan mucho más de las casas de estudio superiores que la formación de sus hijos e hijas.

Hasta los últimos años, cuando el estado (a través de sus procesos de aseguramiento de la calidad y de los marcos legales de la reforma educacional iniciada en el año 2016) instala la noción de Vínculo con el Medio (COMISIÓN NACIONAL DE ACREDITACIÓN, 2015) como dimensión de acreditación que contribuye significativamente al financiamiento de la universidad, ésta empieza a mirar de un modo diferente el territorio en el que se encuentra. Ya no sólo como un terreno del cual se pueden extraer postulantes y matrículas y en el cual se debe abonar con proyectos o programas para asegurar su enraizamiento predatorio en él. Nuevamente, como ha sido desde el cambio del sistema universitario de 1981, hacia un sistema educacional competitivo y de mercado, será el acceso y competencia por el dinero lo que ordenará la orientación y los valores superiores coyunturales de las universidades públicas. Parece un absurdo, pero en un sistema neoliberal económico, político aún con restricciones, educación de mercado, la educación superior no puede desarrollarse en plenitud como institución de la sociedad para el servicio de las mayorías y de su desarrollo. 
La Universidad de Inicios del Siglo XXI: el compromiso de la universidad consigo misma: Extensión Universitaria, Responsabilidad Social Universitaria, Vínculo con el Medio.

Actualmente hay varios conceptos que se refieren a las relaciones entre las universidades y el territorio. Antes, es necesario reconocer que la noción de "territorio" puede resultar engañosa si es leída desde una lógica reproductivista del sistema económico neoliberal e ideológico neoconservador. ¿Es en sí la relación universidad-medio un vínculo virtuoso "per se"? No necesariamente, ya que este vínculo puede ser un modo de fortalecer el sistema-ambiente que ha llevado a la universidad a cumplir una función adaptativa más que transformadora. Es la concepción política de la educación y de la universidad la que determina si el vínculo es un proceso de trasvasije de saberes que no modifican las condiciones que determinan el sentido y la misión de la universidad o es un proceso que intenta contribuir a la transformación del territorio como condición de su propia renovación, de una reconstrucción del modo en que establece los canales de comunicación crítica con la sociedad y el proyecto de desarrollo territorial orientado a los intereses de las mayorías, en términos de inclusión, justicia social, participación, desarrollo y democracia.

Brandao (2007) plantea que el sistema capitalista profundiza y complejiza la división social del trabajo, en todas las dimensiones, inclusive en la espacial; por ello los conceptos que tratan de relaciones entre actores o instituciones en su interior, se significan en cada lugar y momento histórico particular. Las relaciones entre universidad y territorio se pueden explicar en ese contexto y deben ser consideradas en los tiempos y las diversas escalas en que el capitalismo globalizado opera: ni la planetaria ni la local son en sí mismas escalas independientes, son interdependientes. Por eso, para la universidad, de modo permanente, es necesario repactar sus relaciones tradicionales y consolidadas con la sociedad, reconstruir espacios públicos y canales institucionales de concertación de intereses entre la academia y los actores sociales del espacio con el que interactúa o quiere relacionarse, establecer contratos sociales territorializados para construir saberes integrados y procesos pedagógicos o políticos de desarrollo, generar espacios de construcción colectiva de conocimiento a través de metodologías participativas o cooperativas. Ello requiere deconstruir las relaciones establecidas en el contexto de una universidad autoritaria, de un pensamiento neoconservador propio del modelo hegemónico económico neoliberal y cerrada en sí misma, para construir una nueva relación en que la comunidad de actores de la sociedad (a la escala que sea) pueda participar de la construcción de la universidad, de su misión, cuanto de los procesos de producción de conocimiento, al menos al nivel de investigaciones aplicadas y de orientación de la ciencia básica. 
Paulo Freire, en Extensão ou Comunicação? (1988), se adelanta casi 40 años a esta preocupación actual al hacer una gran crítica al concepto de Extensión, en ese momento agrícola, pero que se puede transferir, conceptualmente, al de extensión universitaria (EU). La extensión es un modo en que el conocimiento producido en una unidad de producción de conocimiento del sistema social (en este caso la universidad) se traspasa - a través de los profesionales y técnicos - a los sectores populares, campesinos, trabajadores, empresarios como si fueran una verdad comprobada, diríamos una verdad "verdadera" en el lenguaje popular y no una "posverdad" en el lenguaje de Oxford, sin que los "objetos" de aprendizaje y aplicación del conocimiento que se les entrega puedan ser analizados críticamente, contrastados con sus propios saberes y modificados por éstos a partir de sus propias pautas de conocimiento y sabiduría. La producción del conocimiento como actividad colaborativa empieza en la disciplina y termina en la interdisciplinariedad, en diálogo más o menos efectivo y en productos de diversos impactos en los fundamentos de las ciencias o en resultados tecnológicos, pero raramente avanza a una concepción intertransdisciplinaria (PADILHA, 2004) - en un sentido científico y pedagógico que avance desde la cooperación entre saberes de diversas disciplinas con el saber de las comunidades.

Hoy en el capitalismo esta relación unidireccional autoritaria se esconde bajo el eufemismo de una relación entre la oferta de conocimientos, como oportunidades de acceso al conocimiento para consumidores que son "libres" de comprarlos, adherirse o utilizarlos, sin que pueda existir una relación en sentido opuesto e incluso con la dificultad de romper el orden establecido de la relación mercantil que implica una concepción extensionista del vínculo universidad-territorio. Las dudas y opciones que implica asumir la visión opuesta, la de la comunicación, es decir, aquella en que la relación de modificación sustantiva, auténtica, honesta, es bidireccional, no dejan ser también políticas y afectan particularmente a los académicos y profesionales que se hacen responsables, desde su formación de pregrado y posteriormente de posgrado, de transmitir - a través de proyectos o procesos de capacitación y educación continua - los saberes aprendidos en su paso por la universidad, que no han sido, en general, aquellos de los sujetos o territorios, en los que se desempeñará:

Se ha profundizado, en el contexto neoliberal, la distancia entre el saber popular e indígena y el saber dominante y validado por el mercado y los grupos, clases o etnias dominantes. En la interfaz de la relación, se encuentran los(as) profesionales que deben enfrentar hoy la misma pregunta: ¿se actúa extendiendo el conocimiento dominante o hegemónico al pueblo o comunidades, se coloca en el lado del saber de los(as) oprimidos(as) - que no es subordinado ni dominado, sino validado por el propio pueblo, con sus contradicciones y debilidades, al igual que el saber dominante o hegemónico - o se actúa promoviendo el diálogo creativo y productivo entre ambos saberes? (WILLIAMSON, 2013, p. 51). 
Esta noción unidireccional de la formación, de la producción y difusión del conocimiento científico, tecnológico y la innovación no sólo representa la hegemonía de una concepción de ciencia hegemónica desde hace centurias sino implica un menoscabo para la formación de los profesionales y académicos en la medida en que se castra del acceso a una parte del conocimiento humano producido durante milenios en territorios profundos de la historia natural y cultural. El extensionismo - "extendido" a la relación entre la universidad y el territorio, entre la ciencia y sus saberes con el desarrollo de la sociedad a cualquier escala - no sólo expresa una concepción caduca de la relación entre las casas de la ciencia y las casas de la vida, sino un modo de deshumanizar a los profesionales que forma y académicos que investigan y una relación autoritaria de imponer un contenido cultural hegemónico, como verdadero, a las comunidades, su lengua, cultura, cosmovisiones.

La Responsabilidad Social Universitaria (RSU) es otra variante conceptual de esta dimensión de la universidad pública. Ella es una adecuación del concepto de Responsabilidad Social Empresarial (RSE) del mundo de los negocios (también denominada Responsabilidad Social Corporativa): tiene su origen no en un debate interno a la universidad sino en el modo en que los sectores económicos e ideológicos dominantes extienden sus nociones básicas de comprensión del mundo, de la ciencia, el conocimiento y la universidad, imponiendo sus marcos ideológicos - a través del estado (Ministerio de Educación, leyes y reglamentos) -, generando situaciones conceptuales complejas y contradictorias: ¿podría alguien pensar que es necesario generar un quehacer específico de responsabilidad social en una universidad pública y estatal? Hacer la pregunta ya es un absurdo, sin embargo en el sistema de educación neoliberal la respuesta es: sí. Es tal el abismo que la educación de mercado ha creado entre la producción científica, el curriculum de formación de profesionales y el desarrollo del territorio, del mundo real de las mayorías, de los desafíos al conocimiento de las comunidades, que ha sido necesario extrapolar un concepto empresarial para responder a esta carencia esencial. En la empresa capitalista privada no es un absurdo ya que la relación entre ella y el mercado es directa: la transacción de bienes y servicios, por dinero, con los consumidores (individuales, empresas, estado) para satisfacer necesidades reales o creadas y aumentar la rentabilidad del capital.

Por ello es que es necesario integrar desde la formación inicial prácticas formativas asociadas al trabajo voluntario y generoso de los estudiantes que permitan efectivamente prestar un servicio a las comunidades, dialogar con éstas, formarse críticamente y modificar la conciencia propia respecto de la disciplina como del quehacer profesional, fuera de la lógica de mercado, (WILLIAMSON et al., 2015) es decir, se podrá considerar este proceso desde una dimensión pedagógica, formativa, en cuanto experiencia de formación ciudadana para cumplir el rol social de la uni- 
versidad. Ello supone cuestionar la mirada dicotómica de lo curricular en relación a lo extracurricular como si fuesen partes de procesos formativos paralelos, pero también implica negar la separación entre teoría y práctica (DÁVILA, 2018) que es una segunda distinción que contribuye a distanciar la universidad de las comunidades en que se inserta y del desarrollo del país y sus regiones. Son estas maneras de establecer una relación con el territorio, la del servicio voluntario con aprendizaje, asociado al curriculum de formación, integrando todas las experiencias de aprendizaje, haciendo indivisible en la práctica social la dicotomía teoría y práctica y generando conciencia crítica en los futuros profesionales, los que pueden otorgarle al concepto de RSU al menos una connotación propia y particular más académica respecto de la RSE que puede tener muchas acepciones que no discutiremos aquí, desde una "sincera" de compromiso con el territorio y sus comunidades, una de mitigación de impactos, una de "marketing" para ocultar una imagen que inhiba conflictos con el territorio o una que aumente el consumo de ciertos sectores de consumidores. La RSU desde este enfoque puede romper el absurdo de su definición, como si fuese posible que una universidad estatal no tuviera esa responsabilidad... ¿y si fuera así? ¿no es lo que estamos discutiendo aquí?

Quizás la RSU sea justamente la expresión de que la universidad ha sido irresponsable en su servicio a los territorios respecto de los requerimientos de sus intereses políticos y sociales, sus necesidades de sobrevivencia, interculturalidad, de tierra, agua, aire limpio, trabajo, superación de la pobreza y problemas sociales como el desempleo o el tráfico y consumo de drogas, defensa ante la hegemonía cultural y lingüística global, en fin: ante el mundo de las personas, organizaciones, comunidades que exigen conocimientos y saberes para contribuir a superar sus propias condicionantes limitantes de la expansión de sus potencialidades. Sin duda que hoy más que nunca se requiere un debate sobre la universidad pública y estatal en relación al desarrollo de los territorios a partir de una crítica al porque ha sido necesario asumir el concepto empresarial de RSE para la universidad estatal y pública cuya misión más profunda y original, aunque, por cierto no la única importante (la producción científica, de las artes y humanidades en sí mismas, de modo autónomo y autosuficiente es igualmente central en la misión de la universidad) es justamente servir a la sociedad a la que se debe, la que le ha dado origen y financia, que es propietaria a través del estado de su institucionalidad y materialidad. La RSU en la universidad estatal, como concepto y práctica, es un síntoma de como la privatización y el mercado se han introducido subrepticiamente en el ámbito público de la educación, despolitizándola y desestatizándola, haciéndola menos pública, independientemente de leyes que permitan ampliar el acceso, democratizar el ingreso a sus aulas. Aunque ese proceso puede contar con el apoyo del estado para fortalecer la privatización debilitando lo público al promo- 
ver la RSU como expresión necesaria para justificar lo que queda de público en el sistema universitario. Hay indicadores - que no trataremos aquí - que muestran esta privatización, por ejemplo, proyectos de incubadoras de negocios (acuerdos de financiamiento entre gobierno regional, empresas y universidades) que muestran que los principales beneficiados de los proyectos no han sido trabajadores, innovadores en negocios de baja cantidad de capital o conocimiento, del sector cooperativo o autogestionario, sino jóvenes profesionales empresarios, muchos con posgrado y vinculados a grupos familiares con negocios mayores. La influencia de académicos es muy baja en la gestión política del estado o en la construcción de políticas, menos en programas de transformación social.

En los últimos años, desde el estado y en el contexto de los procesos de aseguramiento de la calidad de la educación se ha empezado a construir, aplicar y reglamentar un concepto más próximo a lo que subyace en este texto: una relación bidireccional y cooperativa entre universidad y territorio, en el cual éste se beneficia de los saberes producidos por el sistema de educación superior el que, a su vez, lo hace del saber instalado en la memoria histórica de los pueblos, comunidades o territorios: el Vínculo con el Medio. Sin embargo no debería ser "vínculo con..." ya que si la universidad se sintiera parte constituyente del territorio y éste la reconociera como "uno más de quienes lo conforman", no se necesitaría usar vínculo (enlace, unión, atadura) sino cooperación, pero por ahora, asumamos el concepto que empieza a instalarse en el medio y sistema universitario y educacional chileno.

Von Baer (2009) hace un análisis respecto del vínculo con el medio en el que propone transitar de lo que denomina el "modo tradicional" de relaciones con lo que denomina "el entorno" a un "modo bidireccional" de articulación. Von Baer coloca la relación en el espacio de la gestión, con consecuencias en los aspectos propiamente académicos de la vida universitaria:

En este contexto, postulamos que la nueva forma en que las universidades se han vinculado con su medio debiera transitar desde un "modo tradicional", caracterizado principalmente por una relación unidireccional desde la universidad hacia un medio compuesto por grupos e instituciones acotadas, a un "modo bidireccional", donde el medio, compuesto ahora por grupos de interés más amplios, debe ser incorporado en el transversalmente en la definición estratégica y funcionamiento de la universidad. Visto así, la vinculación con el medio se constituye hoy como un desafío institucional fundamental para la gestión de las universidades, que determina no sólo su éxito como organización, sino que constituye una dimensión clave del desarrollo del país en la sociedad del conocimiento (2009, p. 455).

Luego plantea pasar de la universidad "torre de marfil" a la "interactiva": se trata de sustituir lo que nos queda de "torre de marfil", por un modo de "universidad interactiva", como mejor forma de servir los intereses de la propia universidad como los de la sociedad en la que está inserta y a cuyo desarrollo y bienestar se debe. Además, es el mejor camino para legitimarse y mantener vigencia como institución fundamental de una sociedad cada vez más compleja y en permanente proceso de cambios (2009, p. 463). 
Señala que es necesario considerar el medio interno: orientado a su propia esencia, que es la producción de conocimiento y formación de personas; y medio externo que es el que la justifica. Ante este último la relación es unidireccional: de la institución a la sociedad sin retroalimentación efectiva. Indica la importancia de la interacción bidireccional en las relaciones, la generación de centros de pensamiento regionales, la prestación de cuentas como contraparte de la universidad a los recursos entregados por la sociedad.

El Vínculo con el Medio, en Chile, se empieza a constituir en una dimensión clave para la acreditación cualitativa de las universidades, pero, también, para su financiamiento, ya que se constituye en uno de los factores que las nuevas leyes de educación superior y universidades estatales (a la fecha de este trabajo esperando su promulgación por el ejecutivo) considerarán como relevantes para la asignación de recursos públicos.

\section{Vínculo con el medio en Chile: la institucionalidad en instalación}

La Comisión Nacional de Acreditación (CNA-Chile) es un ente público y autónomo que tiene como función la de verificar y promover la calidad de las universidades, institutos profesionales y centros de formación técnica autónomos, y de las carreras y programas que estos imparten. Tiene como contexto legal la Ley que consagra un Sistema Nacional de Aseguramiento de la Calidad de la Educación Superior ( $\mathrm{n}^{\circ}$ 20.129) (CHILE, 2006), promulgada en octubre de 2006. A partir de esta Ley y otras reglamentaciones subsecuentes, el estado ha ido asumiendo un rol más activo en la regulación y control de la calidad de la educación superior, en un contexto de educación de mercado neoliberal, lo que ha significado una pérdida de espacios de acreditación a las agencias privadas en esta función y la monopolización por el estado de algunas disciplinas como las carreras de pedagogías y de la salud a nivel de pregrado (formación profesional inicial).

En Chile, los lineamientos del sistema de acreditación y aseguramiento de la calidad en instituciones de educación superior, le han dado mayor importancia a los distintos modos de Vinculación con el Medio. La distancia entre los desafíos del desarrollo y crecimiento nacional, las demandas sociales por soluciones a problemas ambientales, sociales, económicos, políticos con la universidad ha llegado a un punto en que la sociedad, algunos sectores de la comunidad académica y científica, los movimientos estudiantiles y el gobierno (de centro-izquierda) consideró que era urgente de abordar. A fines del año 2016, 37 universidades (públicas y privadas) lograron acreditarse en esta dimensión (COMISIÓN NACIONAL DE ACREDITACIÓN, 2016) promediando 4,7 años de acreditación sobre un máximo de 7.0. 
Las funciones de la CNA-Chile son tres:

1. acreditación institucional;

2. autorización de agencias acreditadoras (privadas);

3. acreditación de carreras de pregrado y programas de postgrado.

Se debe señalar que la acreditación institucional tiene como principal propósito la evaluación de los procedimientos y mecanismos de autorregulación de la calidad que tienen las instituciones de educación superior. Esta orientación da cuenta de que la responsabilidad primera es de la propia institución y no de algún órgano externo a ella y se parte de la premisa de que dichas instituciones cuentan con normativas y políticas claras y coherentes, operacionalizadas por procedimientos y reglamentos que permitan dicha autorregulación en sus procesos y resultados, en pos de lograr los fines y objetivos institucionales establecidos en su misión y visión. Si bien una lectura de este enfoque es el de la falta de regulación estatal que favorece la privatización del sector, para las universidades estatales es condición de su autonomía, pluralidad, libertad de cátedra.

Para cumplir con los procesos de evaluación, las dimensiones básicas o mínimas consideradas por la CNA-Chile para acreditar a las instituciones de educación superior son las de Gestión Institucional y la Docencia de Pregrado, siendo estas las más esenciales y obligatorias desde el punto de vista de los destinatarios principales de su quehacer: los estudiantes, futuros profesionales. Es obligación para dictar carreras del área de la Pedagogía y Salud el que ellas estén acreditadas.

El proceso de autoevaluación y evaluación externa sobre la calidad de sus actividades, considera los siguientes ítems:

- una autoevaluación interna;

- una evaluación externa;

- la decisión de acreditación.

Se mencionaba que la gestión institucional y la docencia de pregrado son áreas mínimas de acreditación exigibles por la institucionalidad; por lo que las áreas adicionales a estos procesos son la de docencia de postgrado, la investigación y la Vinculación con el Medio.

Según la CNA-Chile (2016), la Vinculación con el Medio se refiere a una serie de nexos y redes de articulación con el medio afín a lo disciplinario, a lo artístico, lo tecnológico, lo productivo o profesional; con el propósito de realizar mejoras en las funciones institucionales, académicas y profesionales de la comunidad universitaria, permitiendo de esta forma alcanzar los objetivos estratégicos definidos en sus planificaciones, tanto la institucional, como la de las macrounidades y unidades que la componen. Para el caso de la Vinculación con el Medio, los requerimientos 
para alcanzar la acreditación institucional en universidades se desglosan principalmente en los siguientes criterios:

- diseño y aplicación de una política institucional de Vinculación con el Medio, que incluya al menos la identificación del medio externo relevante y la utilización de los resultados de la vinculación para el mejoramiento de la actividad institucional;

- instancias y mecanismos formales y sistemáticos de Vinculación con el Medio externo;

- asignación de recursos suficientes para asegurar el desarrollo de actividades de Vinculación con el Medio;

- vinculación de estas actividades con las funciones de docencia de pre o postgrado, o con las actividades de investigación cuando corresponda;

- impacto de la Vinculación con el Medio en la institución y en el medio externo.

\section{Articulación inter-institucional: la red de vinculación con el medio de las universidades estatales de Chile}

Desde hace unos años, con la idea de constituirse como un espacio de encuentro e intercambio de experiencias y prácticas institucionales, las universidades estatales de Chile han concretado el Consorcio de Universidades del Estado de Chile (CUECh), el cual a su vez, ha establecido redes en su interior para abordar las distintas funciones universitarias:

- red de coordinadores institucionales;

- red de pregrado;

- red de aseguramiento de la calidad;

- red de gestión económica y administrativa;

- red de análisis institucional;

- red de investigación;

- red de arte, cultura y patrimonio;

- red de directores de comunicación;

- red de vinculación con el medio.

Para esta última, el grupo de trabajo ha desarrollado su propia conceptualización sobre la Vinculación con el Medio, proponiéndose una definición consensuada 
que represente la mirada de quienes integran esta red. La resultante conceptual es la siguiente:

La Vinculación con el Medio es una función esencial, expresión del irrenunciable compromiso público de las Universidades Estatales que se manifiesta en un sistema integrado de relaciones transdisciplinares con su entorno significante, y que apunta a la construcción compartida de bienes públicos para el desarrollo sostenible a nivel regional y el fortalecimiento de la investigación, la docencia, la extensión y comunicación universitaria, de tal manera de consolidarse como un referente social y cultural a nivel nacional e internacional (RED DE VINCULACIÓN CON EL MEDIO, 2017, p. 6).

Es interesante como la red de universidades estatales establece la transdisciplinariedad (y no la interdisciplinariedad) como el concepto que articula el saber universitario con el saber del entorno; ello expresa una nueva visión respecto de esta dimensión, particularmente en el campo del conocimiento que es la esencia de la universidad (como producción y difusión, para una formación de personas de calidad, críticas y con compromiso social) y no sólo en actividades esporádicas o publicitarias.

La Red ha relevado la función de la Vinculación con el Medio a través de distintas dimensiones:

- la institucionalidad de la vinculación con el medio;

- la construcción compartida de bienes públicos;

- la pertinencia de los procesos académicos e institucionales con el desarrollo sostenible de la región;

- la ética en las relaciones interinstitucionales e intersectoriales.

\section{Conclusiones}

La Vinculación con el Medio se ha transformado últimamente en una de las áreas universitarias que se han visto fortalecidas y relevadas en el rol que las universidades cumplen en la sociedad desde que estas han sido creadas; empujadas por las distintas transformaciones socioculturales y económicas, las universidades han derivado en nuevas funciones y propósitos, como lo es el posicionamiento y proyección, la transferencia tecnológica, la formación continua, las prestaciones de servicios y consultorías, y también las relaciones internacionales. Lo anterior, como complemento y valor agregado a las tradicionales actividades de lo que hasta ahora se denominaba extensión universitaria.

La lógica de la Vinculación con el Medio de las universidades, es una lógica dialéctica, presente también en procesos de desarrollo local y territorial, en cuanto lo que busca es la articulación, coordinación y colaboración entre distintos agentes 
del desarrollo presentes en los territorios, que tengan como propósito común el dar mejor bienestar a sus habitantes y comunidades, ampliando las oportunidades personales, familiares y laborales de quienes los habitan.

La Vinculación con el Medio también es un aporte al capital social, toda vez que constituye alianzas y redes basadas en la confianza, la reciprocidad, la cooperación, la solidaridad, el voluntariado y la colaboración entre agentes, instituciones y personas que trabajan en pos de un propósito común en una determinado territorio.

A pesar de dichos avances, la vinculación se ha transformado solo en una herramienta indicativa y no normativa, hasta ahora, muy débil como mecanismo de rendición de cuentas de la gestión realizada por académicos, directivos e investigadores universitarios, aunque se encuentran presentes en diversos planes y estrategias de universidades, facultades y otras unidades.

La noción de vínculo con el medio que la institucionalidad chilena le ha dado recientemente a esta área de la educación superior debe orientarse a una mirada descentralizadora y territorial, dado que la interacción con instituciones, organizaciones y comunidades no puede estandarizarse y homogeneizar parámetros e indicadores para todas las universidades y regiones del país debido a la diversidad y heterogeneidad de las dinámicas territoriales presentes. De esta forma, los impactos de la Vinculación con el Medio serán también distintos y diferentes según donde y como interactúe la universidad con un entorno específico. Se busca, por lo tanto que la ella deje de ser unidireccional, en la lógica de extensión universitaria, sino que a lo menos esta interacción sea bidireccional, de reciprocidad, colaborativa y conjunta; que sea multidireccional, entendiendo que las dimensiones del quehacer universitario no se ejercen en un solo ámbito del desarrollo sino que en varios de ellos en forma complementaria y en co-gestión.

Con respecto a la institucionalidad chilena en el ámbito de la Vinculación con el Medio, ésta no ha podido consolidar instrumentos o mecanismos de medición de su impacto en el territorio, que vele por desarrollar actividades e iniciativas con sentido y no solo la ejecución de actividades dispersas y fuera de una lógica de trabajo mancomunado con las comunidades y organizaciones, evitando de esta forma su desarrollo apenas como la suma de pequeños proyectos o iniciativas de corto plazo, a veces individuales, sin continuidad en el tiempo o territorio (cosismo universitario). En lo que se pueden evidenciar algunos avances es en la generación de instancias colaborativas entre las instituciones para intercambiar experiencias y trabajo conjunto, aún en esta etapa inicial de institucionalización y desarrollo.

Medir o conocer el impacto de las acciones de vinculación de las universidades sigue siendo un gran desafío para las casas de estudios, en cuanto a poder identi- 
ficar áreas que no han sido abordadas por las disciplinas y otras de las cuales aún no han respondido satisfactoriamente desde las necesidades y requerimientos de la sociedad local y regional. Pero también, es necesario reconocer que hay universidades que omiten entrar en temas de complejidad epistemológica o política, como es, por ejemplo, la cuestión ambiental, indígena, de afrodescendientes, migrantes o refugiados transnacionales, de economías alternativas al modelo capitalista dominante o de tipo social.

La política de Vínculo con el Medio es resultante de un acuerdo político entre sectores conservadores y el estado en un gobierno progresista. Los primeros buscaron evitar en lo posible un rol activo del estado defendiendo el sector privado del sistema universitario, la libertad de enseñanza y el derecho de los padres a elegir la educación de sus hijos en vez de afirmar el derecho de todos a la educación, la oposición a la gratuidad de la educación y la defensa a un financiamiento a través de "voucher" por estudiante vulnerable. Los segundos querían más regulación estatal, desarrollo de la educación pública, gratuidad y no discriminación, un sistema estatal que poco a poco recobrara su papel central en el sistema educacional y sobretodo el compromiso de las universidades estatales con el territorio en el que se encuentran. Por tanto, habrá que ver en el tiempo las consecuencias de este acuerdo obligado dados los quórums necesarios para ciertas decisiones legislativas y la transversalidad de las posiciones conservadores y progresistas en las diversas alianzas del Congreso Nacional.

Finalmente, se sostiene que la Vinculación con el Medio se debe transformar en un "sensor" que permanentemente monitoree y observe las problemáticas y fenómenos de la sociedad. Lo anterior, con el fin de que la docencia, la investigación y la Vinculación con el Medio, asuman dichas demandas como un insumo clave para reformular planes de estudios de pre y postgrado, procesos de prácticas, nuevas investigaciones y publicaciones. Que permitan a las nuevas generaciones de profesionales enfrentar con mayor éxito y responsabilidad las tareas del desarrollo. Producto de esto, es la generación de una constante retroalimentación en todas las dimensiones del desarrollo y las respectivas disciplinas. Aún es muy difuso este desafío, como para que permita permear los planes de estudios según las experiencias de Vinculación con el Medio, tanto de académicos como de estudiantes, ya que no existen metodologías ni procedimientos definidos o consensuados válidamente para ese objetivo.

Habrá que dar un tiempo aún para conocer los resultados e impactos de esta nueva conceptualización e institucionalidad en el sistema universitario para abordar sus relaciones con el territorio. ¿Superará una noción de extensión universitaria (EU) o de RSU como las describíamos y analizábamos más arriba? No lo 
sabemos aún. Hoy lo que se verifica es que el estado está orientando a las universidades a valorar y comprometerse en su relación con el territorio y, en una lógica de educación de mercado, está colocando incentivos financieros y obligaciones de acciones en el territorio como condición para recibir los aportes del estado a las instituciones, al integrar la Vinculación con el Medio entre los requisitos y las fórmulas de cálculo para el financiamiento estatal a las universidades estatales. Los cambios exigidos no son sólo de las instituciones, implican cambios culturales en los académicos, valoración efectiva de la investigación + desarrollo en un entorno que incentiva monetariamente y financia la educación según productividad académica medida por publicaciones indexadas y proyectos ganados en concursos competitivos, donde a los académicos que trabajan en esta dimensión se les considera como en una segunda categoría. Los cambios deben ser sustanciales, caso contrario esta nueva conceptualización será una moda más y un concepto que se agrega al de extensión y RSU. Pero lo más importante a observar no es lo institucional, sino el modo en que el conocimiento producido en el territorio, a lo largo de la historia y que se acumula en la memoria cognitiva, cultural, corporal y patrimonial del territorio y de las formas de vida en ellas impregna, permea, dialoga y construye el conocimiento bajo una epistemología intertranscultural, intertransdisciplinaria. Si el Vínculo con el Medio no es capaz de modificar la misión y democratización de la universidad, los modos de producir conocimientos, los conocimientos en sí mismos y consecuentemente los saberes elaborados, no es posible referirse a la Vinculación con el Medio de un modo pleno y podrá llegar a ser, con sus ventajas y desventajas un concepto o una sigla más que se agrega a la EU, la RSU: ahora el VCM. Habremos dado otro engañoso paso más de retroceso al periodo anterior a la reforma universitaria de Córdoba en Argentina (1918) o a la Reforma Universitaria de Chile (1967).

\section{Notas}

1 Canción "Me gustan los Estudiantes", de Violeta Parra. Disponible en: <https://www.youtube.com/ watch?v=sfOMD_GdeS4>. Acceso en: 22 jul. 2018.

2 Estos proyectos, generados desde el año 2000 a la fecha los ha financiado el Ministerio de Educación, la cooperación internacional, Corporación Nacional de Desarrollo Indígena, Fundación Integra, Ministerio del Medio Ambiente, Empresas nacionales e internacionales, Redes Pedagógicas de Profesores, la Universidad de La Frontera (UFRO) entre otras instituciones. Se han impulsado como proyectos de investigación, investigación acción participativa, investigación en sala de clases e investigación + desarrollo, extensión académica, perfeccionamiento de educadores y profesionales del desarrollo local y regional. Todos coordinados por el primer autor del artículo. Dado el gran volumen de acciones de estas casi dos décadas no listamos todos los proyectos o acciones en la UFRO. Destacamos el Proyecto de Extensión Kelluwün: educación, desarrollo e interculturalidad desde el año 2000, a través del cual agradecemos el importante apoyo de los Proyectos de Extensión Permanente de la Dirección de Vínculo con el Medio de la UFRO; el proyecto de investigación Metodología de Investigación en Sala de Clases: el impacto en el desarrollo personal y pro- 
fesional de los docentes" (2016-2018) DIUFRO DI16-0046; y el de I+D Proyecto Nuestra Escuela Pregunta Su Opinión-NEPSO, ambos de la Universidad de La Frontera (2005 a la fecha) Proyecto FID08-0001. A ello se agrega la experiencia de co-autor que forma parte del equipo de Vínculo con el Medio de la Facultad de Educación, Ciencias Sociales y Humanidades de la UFRO.

3 El "debe ser" del párrafo es indicativo de la falta de pluralidad ideológica o disciplinaria de algunas universidades privadas en Chile: en una no se enseña marxismo en sociología o economía, en otra no se estudian técnicas de aborto en medicina, en una escuela de leyes no se aprende sobre el divorcio.

\section{Referencias}

BADIOU, Alain. ¿Qué es un pueblo? Santiago, Chile: LOM ediciones, 2014.

BRANDAO, Carlos. Território \& Desenvolvimento: as múltiplas escalas entre o local e o global. Campinas, Brasil: Editora da Unicamp, 2007.

COMISIÓN NACIONAL DE ACREDITACIÓN. Criterios de Evaluación para la Acreditación de Carreras Profesionales, Carreras Profesionales con Licenciatura y Programas de Licenciatura. Santiago, Chile: Comisión Nacional de Acreditación, 2015.

. Guía para la Autoevaluación Interna Acreditación Institucional de Universidades. Santiago, Chile: Comisión Nacional de Acreditación, 2016.

DÁVILA, B. Gianina. Evaluación y propuestas de estudiantes sobre el rol social que cumple la universidad. Demandas por una formación democrática. In: FERRADA, D. (Ed.). Políticas educativas y su impacto en las comunidades. Investigación en educación para la justicia social. Talca, Chile: Ediciones UCM, 2018. p. 137-162.

FREIRE, Paulo. Política e educação: ensaios. 5. ed. São Paulo, Brasil: Cortez, 2001.

Extensão ou comunicação? Rio de Janeiro, Brasil: Paz e Terra, 1988.

JIMÉNEZ, D. L. J. M.; LAGOS, R. F. Nueva Geografía de la Educación Superior y de los Estudiantes. Santiago, Chile: AEqualis, 2011.

CHILE. Ley $n \square$ 20.129. Establece un sistema nacional de aseguramiento de la calidad de la educación superior. Biblioteca del Congreso Nacional de Chile (BCN). 2006. (Última versión del 04 de abril de 2017).

MANIFIESTO Liminar de la Reforma Universitaria del 21 de Junio de 1918. 1918. Disponible en: <http://www.bnm.me.gov.ar/giga1/documentos/EL004733.pdf>. Acceso en: 22 jul. 2018.

MATO, Daniel. Educación Superior y pueblos indígenas y afrodescendientes en América Latina: políticas y prácticas e inclsuión, democratización e interculturalización. Saenz Peña, Argentina: Universidad Nacional de Tres de Febrero, 2017.

NOGUEIRA, Adriano (Org.). Ciência para quem? Formação científica para que? Petrópolis, Brasil: Vozes, 2000.

PADILHA, P. Curriculo intertranscultural. Novos itinerários para a educação. São Paulo, Brasil: Cortez, Instituto Paulo Freire, 2004.

RED DE VINCULACIÓN CON EL MEDIO. Marco de Referencia. Red de Universidades Estatales de Chile. Santiago, Chile, 2017. 
SACKS, O. Diário de Oaxaca. Barcelona: National Geographic Society, 2002.

VON BAER, Heinrich. Vinculación con el Medio: ¿función subalterna o esencial de la universidad? In: CNA-CHILE. Desafíos y perspectivas de la dirección estratégica de las instituciones universitarias. Santiago, Chile: Ediciones CNA-Chile, 2009. p. 453-493.

WILLIAMSON, Guillermo et al. Aprendizaje con servicio voluntario en la formación inicial docente. Estud. pedagóg., United Kingdom, v. 41, n. 2, p. 271-286, 2015.

WILLIAMSON, Guillermo. Is the interculturalisation of Chile's universities a real possibility? Arts and Humanities in Higher Education, Valdivia, Chile, v. 16, n. 1, p 51-65, Feb. 2017.

Paulo Freire: ¿Extensión o Comunicación? Sobre los profesionales y el conocimiento en el (no) diálogo de saberes. Crolar: Critical Reviews on Latin American Research, v. 3, p. 50-52, Apr. 2013. 